\title{
ACAROLOGY
}

\section{Biologia de Acarophenax lacunatus (Cross \& Krantz) (Prostigmata: Acarophenacidae) sobre Tribolium castaneum (Herbst) (Coleoptera: Tenebrionidae) e Cryptolestes ferrugineus (Stephens) (Coleoptera: Cucujidae)}

\author{
Carlos R.F. de Oliveira ${ }^{1}$, Lêda R.A. Faroni ${ }^{2}$, Raul N.C. Guedes ${ }^{1}$, José R. Gonçalves ${ }^{1}$ e Flávia M. \\ GARCIA $^{2}$ \\ 1DDepto. Biologia Animal,Entomologia, crfoliveira@hotmail.com; ${ }^{2}$ Depto. Engenharia Agrícola, Ifaroni@ufv.br \\ Univ. Federal de Viçosa, 36570-000, Viçosa, MG
}

Neotropical Entomology 36(3):459-464 (2007)

Biology of Acarophenax lacunatus (Cross \& Krantz) (Prostigmata: Acarophenacidae) on Tribolium castaneum (Herbst) (Coleoptera: Tenebrionidae) and Cryptolestes ferrugineus (Stephens) (Coleoptera: Cucujidae)

\begin{abstract}
The aim of the present study was to assess the effect of six different temperatures on the development of Acarophenax lacunatus (Cross \& Krantz) using eggs of Tribolium castaneum (Herbst) and Cryptolestes ferrugineus (Stephens) as hosts. The temperature affected the development of $A$. lacunatus. The largest values for the progeny (19 mites in $T$. castaneum and 15 mites in C. ferrugineus) were obtained at about $30^{\circ} \mathrm{C}$, as also observed for the net reproductive rate $\left(\mathrm{R}_{\mathrm{o}}\right)$, which revealed that the $A$. lacunatus population increased 18 times in T. castaneum and 14 times in C. ferrugineus in a generation. The intrinsic rate of increase $\left(\mathrm{r}_{\mathrm{m}}\right)$ gradually increased with temperature, reaching the maximum value at $35^{\circ} \mathrm{C}$ in $T$. castaneum $(1,608)$ and $C$. ferrugineus $(1,289)$. The generation time was negatively correlated with temperature, ranging from 1,60 to 4,85 days in $T$. castaneum and from 1,96 to 5,34 days in C. ferrugineus. These results suggest that the mite A. lacunatus may be used in programs of biological control of $T$. castaneum and $C$. ferrugineus in the tropics.
\end{abstract}

KEY WORDS: Mite, life table, biological control, storage pest

RESUMO - O presente trabalho teve por objetivo avaliar o efeito de seis temperaturas (20, 25, 28, 30, 32 e $35^{\circ} \mathrm{C}$ ) sobre os parâmetros biológicos de Acarophenax lacunatus (Cross \& Krantz) utilizando ovos de Tribolium castaneum (Herbst) e Cryptolestes ferrugineus (Stephens) como hospedeiros. A temperatura influenciou o desenvolvimento do ácaro A. lacunatus. Os maiores valores para a progênie (19 ácaros em $T$. castaneum e 15 ácaros em $C$. ferrugineus) foram obtidos a $30^{\circ} \mathrm{C}$. O mesmo foi observado para a taxa líquida de reprodução $\left(\mathrm{R}_{\mathrm{o}}\right)$, que revelou que a população de $A$. lacunatus aumentou 18 vezes em $T$. castaneum e 14 vezes em C. ferrugineus em uma geração. A taxa intrínseca de crescimento $\left(\mathrm{r}_{\mathrm{m}}\right)$ aumentou com a elevação da temperatura, atingindo o valor máximo a $35^{\circ} \mathrm{C}$ em $T$. castaneum $(1,608) \mathrm{e}$ em C. ferrugineus $(1,289)$. O tempo de geração foi inversamente proporcional à temperatura, variando de 1,60 a 4,85 dias em $T$. castaneum e de 1,96 a 5,34 dias em $C$. ferrugineus. Esses resultados sugerem que o ácaro A. lacunatus pode ser utilizado em programas de controle biológico de T. castaneum e de C. ferrugineus em regiões tropicais.

PALAVRAS-CHAVE: Ácaro, tabela de vida, controle biológico, praga de grãos armazenados

Os membros da família Acarophenacidae (Acari: Prostigmata) são parasitas de ovos de várias espécies de insetos e estão distribuídos em sete gêneros: Protophenax, Aethiophenax, Aegiptophenax, Paracarophenax, Acarophenax, Adactylidium e Paradactylidium (Magowski 1994, Kaliszweski et al. 1995, Goldarazena et al. 2001). Tais ácaros apresentam uma história de vida bem característica, atuando segundo Lindquist (1983), como parasitóides porque: a) sua atividade de alimentação resulta na destruição do ovo hospedeiro; b) apenas um ovo hospedeiro é requerido para o desenvolvimento da progênie; c) somente um estágio (neste caso a fêmea adulta) ataca o hospedeiro; d) a fêmea adulta é quem dispersa e localiza o hospedeiro para as próximas gerações.

Alguns desses ácaros estão ecologicamente associados a diversas famílias de coleópteros (Cerambycidae, Nitidulidae, Scolytidae, Dermestidae, Cucujidae, Tenebrionidae e Bostrichidae), de trips (Phlaeothripidae) e de dípteros (Sciaridae) (Goldarazena et al. 1997, Khaustov 1999, Oliveira 
et al. 2002). Entretanto, a associação com hospedeiros não é conhecida para Paradactylidium e Aegiptophenax (Rady 1992, Goldarazena et al. 1999). Existem poucos estudos sobre a biologia das espécies da família Acarophenacidae, os quais estão limitados às pesquisas realizadas por Rack (1959), Steinkraus \& Cross (1993) e Faroni et al. (2000, 2001).

O gênero Acarophenax contém nove espécies (A. assanovi Livshitz \& Mitrofanov, A. lacunatus (Cross \& Krantz), A. lukoschusi Mahunka \& Fain, A. mahunkai Steinkraus \& Cross, A. makros Ostojá-Starzewski, A. meropsi Rakha \& Kandeel, A. nidicolus Cross \& Krantz, A. rackae Mahunka \& Zaki e A. tribolii Newstead \& Duvall). Machos desse gênero são conhecidos apenas nas espécies A. lacunatus, A. mahunkai e A. tribolii, e foram citados ou descritos por Newstead \& Duvall (1918), Steinkraus \& Cross (1993) e Faroni et al. (2001).

Quatro espécies foram coletadas diretamente de insetos hospedeiros que, com uma exceção, eram todos coleópteros: A. assanovi sobre Rhyzopertha dominica (Fabricius) (Livshitz \& Mitrofanov 1974); A. lacunatus sobre Cryptolestes ferrugineus (Stephens) (Cross \& Krantz 1964); A. mahunkai sobre Alphitobius diaperinus (Panzer) (Steinkraus \& Cross 1993); A. tribolii sobre Tribolium castaneum (Herbst), Tribolium confusum Jacquelin du Val (Newstead \& Duvall 1918), Gnathocerus cornutus Fabricius, Latheticus oryzae Waterhouse, Palorus ratzeburgi (Wissmannn) (Marlatt 1932) e Sciara hirtilineata Brunetti (Wadhi \& Kishore 1975). As outras cinco espécies foram coletadas de vários substratos onde podem ser encontrados insetos: A. lukoschusi de fezes de morcego (Mahunka \& Fain 1989); A. makros de fezes de aves (Gao \& Zou 1994); A. meropsi do pássaro Merops apiaster (Rakha \& Kandeel 1983); A. nidicolus em um ninho do pássaro Colaptes cafer (Cross \& Krantz 1964) e A. rackae em fezes de ovelha (Mahunka \& Zaki 1991).

O primeiro relato do ácaro A. lacunatus foi feito por Cross \& Krantz (1964), que o descreveram a partir de populações de laboratório de C. ferrugineus em Pullman, Washington, USA. Os autores não fornecem dados sobre o comportamento e a biologia das espécies de Acarophenax identificadas (A. lacunatus e $A$. nidicolus), mas comentam serem possíveis parasitas de ovos de coleópteros. Faroni et al. (2000, 2001) estudaram a biologia de A. lacunatus sobre o hospedeiro $R$. dominica em diferentes temperaturas e o potencial de utilização desse ácaro como agente de controle biológico desse coleóptero. Oliveira et al. (2002, 2003a, 2003b) avaliaram a preferência e o potencial de parasitismo de $A$. lacunatus sobre outras espécies de coleópteros, ampliando o número de hospedeiros conhecidos desse ácaro.

A temperatura é um fator ambiental importante que exerce forte influência sobre os parâmetros biológicos dos organismos, agindo de maneira direta ou indireta, sobre a velocidade das diferentes fases do ciclo de vida, no comportamento, nas reações metabólicas, dentre outros (Kogan \& Parra 1981, Debach \& Rosen 1991). Com isso, o tempo de desenvolvimento tanto dos insetos-praga quanto de seus inimigos naturais que vivem em ambientes de grãos armazenados, está sob influência direta da temperatura da massa de grãos (Hagstrum et al. 1996, Faroni et al. 2001).

Dessa forma, é imprescindível o aprofundamento dos estudos básicos sobre A. lacunatus visando sua futura liberação em programas de controle biológico e manejo integrado de pragas em situações de armazenamento de grãos. O objetivo deste trabalho foi avaliar o efeito da temperatura sobre a biologia de $A$. lacunatus alimentado com ovos de $T$. castaneum e C. ferrugineus.

\section{Material e Métodos}

Os coleópteros T. castaneum e C. ferrugineus foram criados em laboratório, em frascos de vidro com capacidade de 1,7 l (17 cm altura x $11 \mathrm{~cm}$ de diâmetro) sobre grãos de trigo semitriturados, com umidade em torno de 13\% (base úmida) (Oliveira et al. 2003b). As populações de A. lacunatus foram obtidas de criações contínuas de $R$. dominica infestadas com o ácaro, nas mesmas condições. Todas as colônias foram mantidas em câmara climatizada com temperatura de $30 \pm 2^{\circ} \mathrm{C}$, UR de 65 $\pm 5 \%$ e escotofase de $24 \mathrm{~h}$. Com base em metodologia de Faroni et al. (2000), as populações de $R$. dominica foram peneiradas visando à obtenção do resíduo de trigo (pó) com ácaros.

Para o estudo da biologia de A. lacunatus, foram coletados 20 ácaros, os quais foram distribuídos em 20 placas de Petri de $5 \mathrm{~cm}$ de diâmetro, contendo 10 ovos (com idade de dois a três dias) de um dos hospedeiros (T. castaneum ou $C$. ferrugineus). Avaliações foram realizadas em intervalos de $1 \mathrm{~h}$ até a fixação do ácaro no ovo do inseto. Quando as fêmeas de A. lacunatus entraram em processo de fisogastria, foram individualizadas em outras placas de mesma dimensão e, até a emergência da progênie, as avaliações passaram a ser realizadas a cada 6h (Oliveira 2005). As variáveis quantificadas foram: 1) o número de indivíduos gerados por fêmea; 2) a razão sexual; 3) o tempo de geração (T); 4) a velocidade de desenvolvimento que é a razão inversa do tempo (1/dias); 5) a taxa líquida de reprodução $\left.\left(\mathrm{R}_{0}\right) ; 6\right)$ a taxa intrínseca de crescimento $\left(\mathrm{r}_{\mathrm{m}}\right)$; 7) o número de gerações/mês de A. lacunatus. Os indivíduos recém-emergidos de cada progênie foram contados e sexados. A sexagem é simples, uma vez que $A$. lacunatus apresenta dimorfismo sexual, como em outras espécies do mesmo gênero, cujo macho é menor que a fêmea, possuindo as pernas IV atrofiadas e opistossoma mais delgado ou afilado (Cross \& Krantz 1964, Steinkraus \& Cross 1993).

Os ensaios para avaliação da influência da temperatura no desenvolvimento e reprodução de A. lacunatus sobre os hospedeiros $T$. castaneum e $C$. ferrugineus foram realizados em câmaras climáticas de temperatura constante a 20, 25, 28, 30,32 e $35^{\circ} \mathrm{C}$ e UR de $65 \pm 5 \%$. A escolha dessas temperaturas se baseou em estudos anteriores com A. lacunatus, sobre o hospedeiro $R$. dominica, que demonstraram que o ácaro não se desenvolve em temperaturas inferiores a $18^{\circ} \mathrm{C}$ e superiores a $40^{\circ} \mathrm{C}$ (Faroni et al. 2000, 2001).

Os parâmetros de tabela de vida foram estimados utilizando-se o software LIFETABLE.SAS desenvolvido por Maia et al. (2000) no ambiente "SAS System" (SAS Institute 1989). Análises de regressão foram realizadas no programa SigmaPlot (Jandel Scientific 1986), sendo que

$$
\begin{aligned}
& \mathrm{R}_{0}=\sum \mathrm{m}_{\mathrm{x}} \cdot \mathrm{l}_{\mathrm{x}} \\
& \mathrm{T}=\sum\left(\mathrm{m}_{\mathrm{x}} \cdot \mathrm{l}_{\mathrm{x}} \cdot \mathrm{x}\right) /\left(\sum \mathrm{m}_{\mathrm{x}} \cdot \mathrm{l}_{\mathrm{x}}\right) \\
& \mathrm{r}_{\mathrm{m}}=\ln \mathrm{R}_{0} / \mathrm{T}
\end{aligned}
$$


Onde:

$r_{m}$ é a taxa intrínseca de crescimento populacional, que corresponde ao número de fêmeas/fêmea/dia; Ro é a taxa líquida reprodutiva, que corresponde ao número de vezes que a população cresce durante o ciclo de vida; T é a duração média de uma geração; $m_{x}$ é o número de descendentes produzidos por fêmea no estágio x (fertilidade específica) e que produzirão fêmeas; $l_{x}$ é a proporção de fêmeas vivas (taxa de sobrevivência) a partir do nascimento até a idade $x ; \mathrm{m}_{\mathrm{x}} \mathrm{l}_{\mathrm{x}}$ é o total de fêmeas produzidas por fêmea durante o intervalo de tempo.

\section{Resultados}

A temperatura influenciou o desenvolvimento do ácaro A. lacunatus criado tanto em T. castaneum quanto em $C$. ferrugineus. A maior taxa de desenvolvimento (1/dia) foi observada a $35^{\circ} \mathrm{C}$ nos dois hospedeiros (Fig. 1).

O número de descendentes de $A$. lacunatus e a razão sexual também foram afetados pela temperatura. Cada fêmea produz uma única geração, uma vez que ocorre sua morte no momento da emergência dos filhos. A maior progênie foi observada para T. castaneum (cerca de 19 indivíduos/fêmea) e para C. ferrugineus (cerca de 15 indivíduos/fêmea) a $30^{\circ} \mathrm{C}$ (Fig. 2). A razão sexual variou de 0,91 a 0,95 em $T$. castaneum e de 0,90 a 0,93 em C. ferrugineus (Fig. 3).

A taxa líquida de reprodução (Ro) indicou que a população de $A$. lacunatus aumentou 18,35 vezes em $T$. castaneum e 13,65 vezes em C. ferrugineus, respectivamente, à temperatura de $30^{\circ} \mathrm{C}$ (Fig. 4).

A taxa intrínseca de crescimento $\left(\mathrm{r}_{\mathrm{m}}\right)$ foi positiva, o que indica que houve crescimento populacional, e aumentou com a elevação da temperatura, sendo maior em ambos os hospedeiros a $35^{\circ} \mathrm{C}(1,608 \mathrm{em} T$. castaneum e 1,289 em $C$. ferrugineus, respectivamente) (Fig. 5).

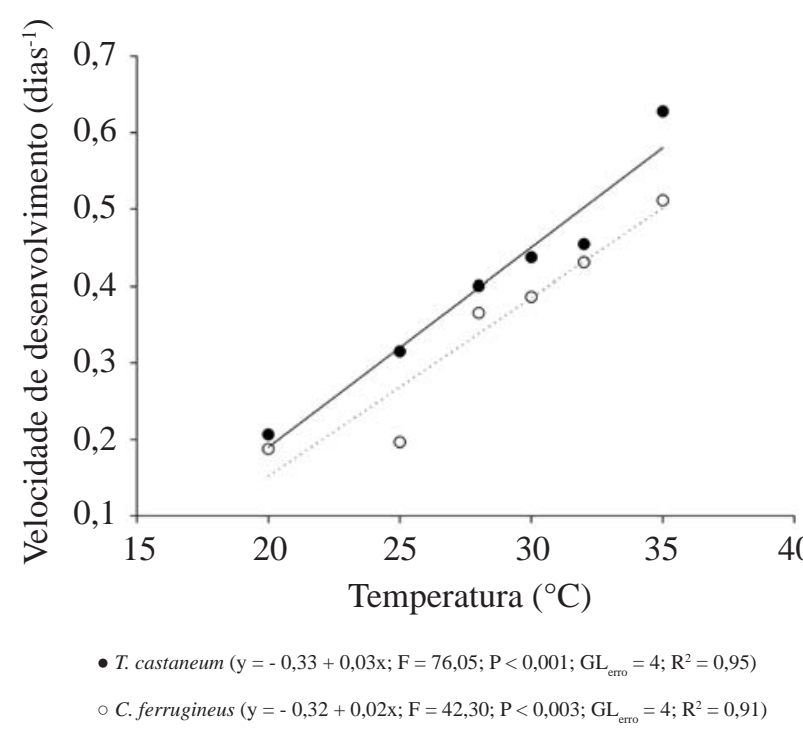

Fig. 1. Efeito da temperatura sobre a velocidade de desenvolvimento de A. lacunatus alimentando-se em ovos de T. castaneum (•) e C. ferrugineus ( $)$ ).
O tempo de geração $(\mathrm{T})$ nos dois hospedeiros foi inversamente proporcional à temperatura (Fig. 6). Por outro lado, o número de gerações/mês do ácaro foi diretamente proporcional ao aumento da temperatura, sendo maior em ambos os hospedeiros a $35^{\circ} \mathrm{C}$.

\section{Discussão}

As altas temperaturas $\left(30^{\circ} \mathrm{C}\right.$ e $\left.32^{\circ} \mathrm{C}\right)$ propiciaram progênie mais numerosa de $A$. lacunatus, que em $T$. castaneum variou de 11 a 19 indivíduos/fêmea enquanto em C. ferrugineus variou de

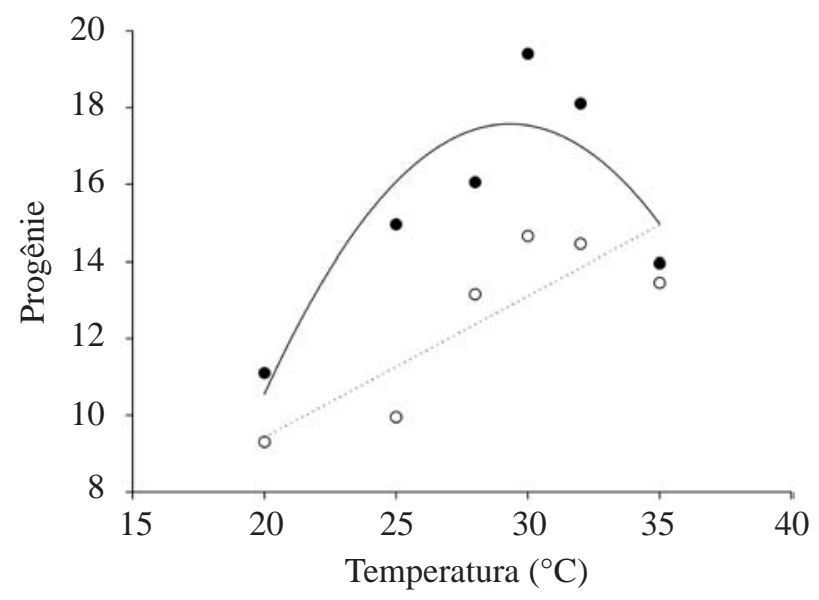

\footnotetext{
- $T$. castaneum ( $\left.\mathrm{y}=-51,84+4,73 \mathrm{x}-0,081 \mathrm{x}^{2} ; \mathrm{F}=49,30 ; \mathrm{P}<0,0001 ; \mathrm{GL}_{\text {erro }}=117 ; \mathrm{R}^{2}=0,46\right)$ $\circ$ C. ferrugineus $\left(\mathrm{y}=-2,06+0,37 \mathrm{x} ; \mathrm{F}=10,42 ; \mathrm{P}<0,03 ; \mathrm{GL}_{\text {eто }}=4 ; \mathrm{R}^{2}=0,72\right)$
}

Fig. 2. Efeito da temperatura sobre a progênie de $A$. lacunatus alimentando-se em ovos de $T$. castaneum $(\bullet)$ e $C$. ferrugineus ( $\mathrm{O}$ ).

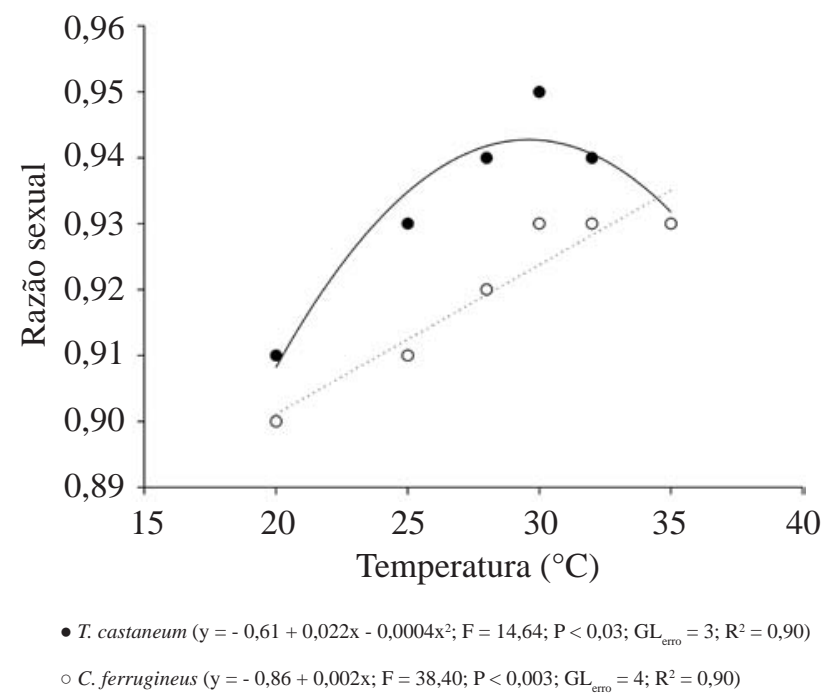

Fig. 3. Efeito da temperatura sobre a razão sexual (n-ôêmeas/ progênie total) de $A$. lacunatus alimentando-se em ovos de $T$. castaneum (•) e C. ferrugineus ( $(\circ)$. 


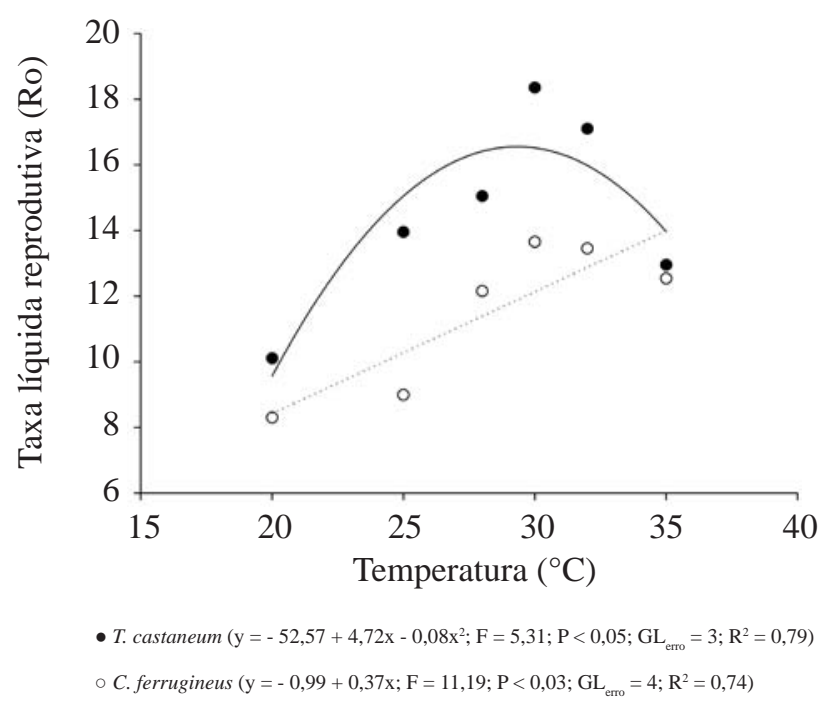

Fig. 4. Efeito da temperatura sobre a taxa líquida reprodutiva (Ro) de A. lacunatus alimentando-se em ovos de T. castaneum $(\bullet)$ e C. ferrugineus (०).

9 a 14 indivíduos/fêmea; foi produzido apenas um macho/fêmea em todas as temperaturas, o que contribuiu para o aumento da razão sexual. Newstead \& Duvall (1918) relatam que uma fềmea fisogástrica de $A$. tribolii produz de quatro a 15 indivíduos e, geralmente, somente um macho. Rack (1959) nunca observou mais que um macho na progênie de Paracarophenax dermestidarum Rack, enquanto três a 27 fêmeas foram produzidas. Já Steinkraus \& Cross (1993) verificaram que de oito a 56 indivíduos/fêmea podem ser produzidos em A. mahunkai, onde até quatro machos foram observados.

Estudando o potencial de parasitismo de A. lacunatus sobre $R$. dominica, Faroni et al. (2000) observaram que a progênie desse ácaro variou de 11 a 17 indivíduos/fêmea, sendo que à temperatura de $30^{\circ} \mathrm{C}$ foi obtido o maior número de indivíduos gerados. Dessa forma, a existência de um ou poucos machos produzidos em Acarophenacidae pode ser suficiente para que todas as fêmeas presentes na progênie sejam fecundadas. Além disso, em termos de custos, a produção de poucos machos é vantajosa, uma vez que as fêmeas é que são encarregadas da dispersão, como comentam Kaliszewski et al. (1995).

A faixa de sobrevivência da maioria das espécies encontra-se entre as temperaturas de $10^{\circ} \mathrm{C}$ e $38^{\circ} \mathrm{C}$. $\mathrm{O}$ aumento da temperatura, até um determinado limite, ocasiona a diminuição da taxa de desenvolvimento e da duração em um estágio em particular (Pedigo \& Zeiss 1996). Faroni et al. (2000, 2001) avaliaram a sobrevivência de A. lacunatus em diferentes temperaturas sobre o hospedeiro $R$. dominica e constataram que esse ácaro não pode se reproduzir nas temperaturas de $18^{\circ} \mathrm{C}$ e $41^{\circ} \mathrm{C}$, e que as condições ótimas para seu desenvolvimento estão próximas a $30^{\circ} \mathrm{C}$. Os autores comentam que altas taxas de desenvolvimento, razão sexual e fertilidade de $A$. lacunatus, em temperaturas superiores a $30^{\circ} \mathrm{C}$, são, provavelmente, fatores que contribuem para a máxima taxa de crescimento populacional do ácaro nessas temperaturas. Isso também foi observado quando

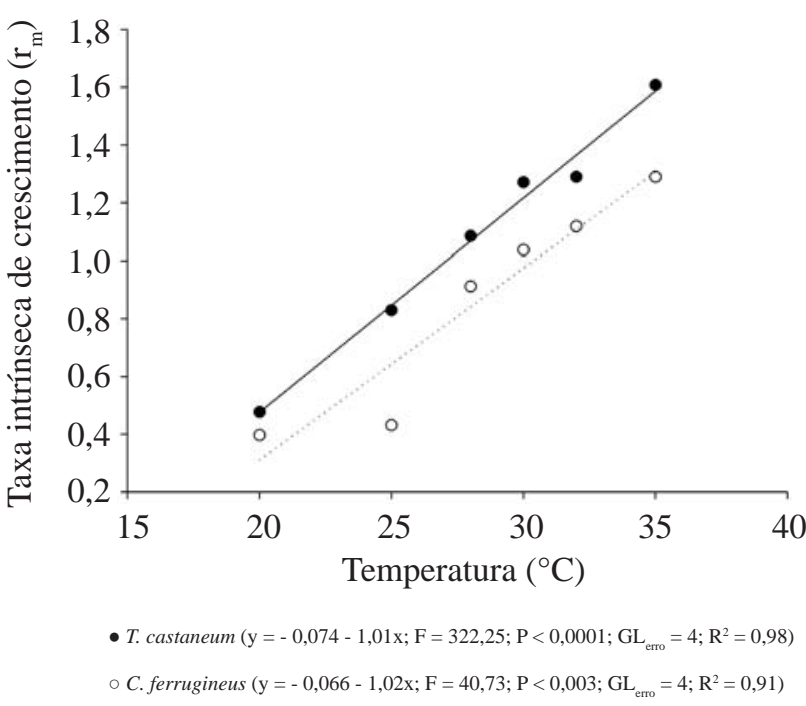

Fig. 5. Efeito da temperatura sobre a taxa intrínseca de crescimento $\left(\mathrm{r}_{\mathrm{m}}\right)$ de A. lacunatus alimentando-se em ovos de $T$. castaneum $(\bullet)$ e $C$. ferrugineus ( $(\circ)$.

A. lacunatus teve T. castaneum e C. ferrugineus como hospedeiros, já que os valores de Ro e $r_{m}$, que expressam o seu potencial biótico aumentaram com a elevação da temperatura. Porém, fica comprovado pelos resultados do presente trabalho que $A$. lacunatus se desenvolve melhor sobre o coleóptero T. castaneum e a implicação disto para o controle biológico é que o ácaro pode apresentar progênie maior. Conseqüentemente, há aspectos positivos relacionados à criação massal de espécies de Acarophenacidae que podem refletir em futuras liberações desse inimigo natural em programas de controle biológico.

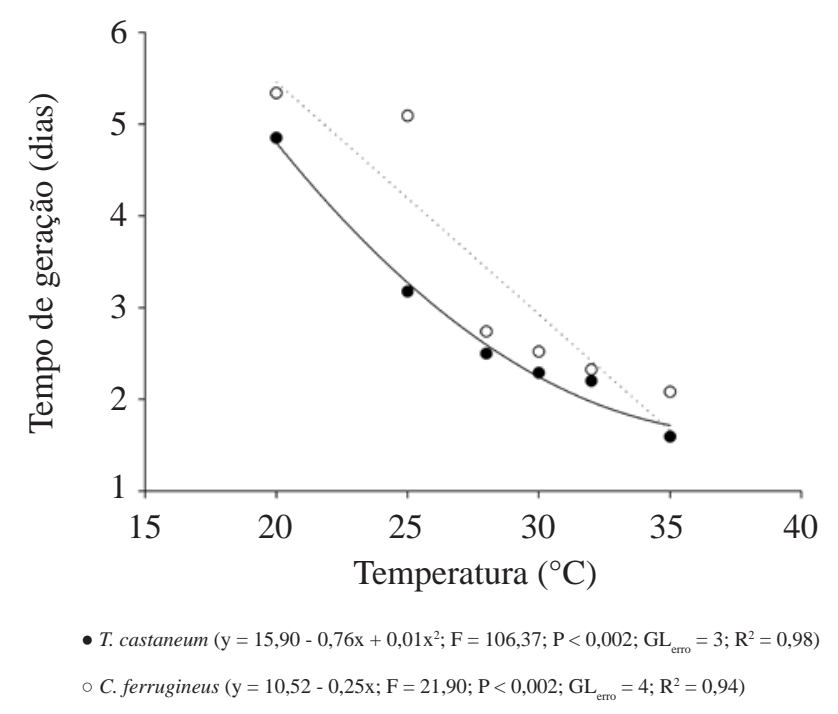

Fig. 6. Efeito da temperatura sobre o tempo médio de uma geração (T) de $A$. lacunatus alimentando-se em ovos de $T$. castaneum (•) e C. ferrugineus ( $($ ). 
No presente estudo, o tempo médio de geração $(\mathrm{T})$ diminuiu com o aumento da temperatura, implicando em um ciclo biológico mais curto em temperaturas mais elevadas. Esse tempo variou quando $A$. lacunatus foi criado nos hospedeiros $T$. castaneum (1,60 a 4,85 dias) e C. ferrugineus (1,96 a 5,34 dias), indicando que a temperatura somada ao tipo e às características do ovo hospedeiro influencia o desenvolvimento desse ácaro. Steinkraus \& Cross (1993) verificaram que o tempo de geração do ácaro A. mahunkai sobre ovos do coleóptero A. diaperinus variou de três a cinco dias, em temperaturas entre $23^{\circ} \mathrm{C}$ e $26^{\circ} \mathrm{C}$. Faroni et al. (2001) observaram que o tempo médio de geração variou de 1,67 a 9,16 dias quando A. lacunatus foi criado no hospedeiro $R$. dominica, o que confirma essas suposições. Em condições de temperaturas mais elevadas, os insetos-praga podem se desenvolver mais rapidamente e suas populações podem aumentar significativamente, daí a razão da relação entre a taxa de desenvolvimento de um inimigo natural e sua presa ou hospedeiro ser crítica para o sucesso do controle biológico (Bernal \& Gonzalez 1993).

Elbadry \& Tawfik (1966) relataram um ciclo de vida muito curto para Adactylidium ficorum Goldarazena \& Ochoa (Acari: Acarophenacidae), com uma nova geração sendo produzida a cada quatro dias $30^{\circ} \mathrm{C}$, o que permite o rápido aumento da população desse ácaro. No presente trabalho, verificou-se que A. lacunatus pode desenvolver até 18 gerações/mês, quando se alimenta de ovos de $T$. castaneum e até 14 gerações/mês quando se alimenta em ovos de $C$. ferrugineus. Resultados semelhantes foram observados por Faroni et al. (2001), que constataram que o potencial biótico de $A$. lacunatus é maior que o de $R$. dominica, sendo então capaz de, num curto período de tempo, desenvolver sua população (12 gerações/mês) de forma superior à desse hospedeiro. Isso também foi verificado por Steinkraus \& Cross (1993), para o ácaro A. mahunkai, que se desenvolveu mais rapidamente que seu hospedeiro, o coleóptero A. diaperinus. Hoschele \& Tanigoshi (1993) também relatam que o ácaro Pyemotes tritici (Lagrèze-Fossat \& Montagné) produziu quatro a cinco gerações durante o intervalo em que Anagasta kuehniella (Zeller) completava uma geração, apresentando, portanto, uma taxa de crescimento também superior à desse hospedeiro. Assim, apresentando um desenvolvimento mais rápido que o dos hospedeiros, os Acarophenacidae podem colonizar o ambiente, exercendo pressão sobre as populações de insetos, mantendo-as em níveis baixos.

À semelhança de outras espécies de Acarophenax, A. lacunatus apresenta muitas características de um agente de controle biológico efetivo, como definido por Bruce (1983) e Lindquist (1983). O ciclo de vida curto permite seu desenvolvimento mais rápido que dos hospedeiros enquanto seu parasitismo ocasiona a destruição dos mesmos. Outro fato importante é que nenhum efeito prejudicial à saúde do homem e de outros animais foi observado por parte de A. lacunatus (Faroni et al. 2000). Sua eficácia na supressão das populações de R. dominica, T. castaneum, C. ferrugineus e Dinoderus minutus (Fabricius) foi demonstrada por Faroni et al. (2000,) e Oliveira et al. (2002, 2003b). Além disso, esse ácaro tem se mostrado tolerante aos principais inseticidas utilizados para o controle de pragas de grãos armazenados (Gonçalves et al. 2004). Assim, esses atributos tornam o ácaro A. lacunatus um inimigo natural eficiente, podendo ser utilizado em programas de controle biológico e/ou manejo integrado de pragas em condições de armazenamento de grãos, principalmente em regiões tropicais.

\section{Agradecimentos}

À Fundação de Amparo à Pesquisa de Minas Gerais (FAPEMIG) e à Coordenação de Aperfeiçoamento de Pessoal de Nível Superior (CAPES) pelo suporte financeiro, e aos revisores pelas valiosas críticas e sugestões.

\section{Referências}

Bernal, J.S. \& D. Gonzalez. 1993. Temperature requires of four parasites of the Russian wheat aphid Diuraphis noxia. Entomol. Exp. Appl. 69: 173-182.

Bruce, W.A. 1983. Mites as biological control agents of stored product pests, p.74-78. In M.A. Hoy, L. Knutson \& G.L. Cunningham (eds.), Biological control of pests by mites. University of California, Berkeley, 185p.

Cross, E.A. \& G.W. Krantz. 1964. Two new species of the genus Acarophenax (Newstead and Duvall, 1918). Acarologia 6: 287-295.

Debach, P. \& D. Rosen. 1991. Biological control by natural enemies. University Press, Cambridge, 2 ed., 386p.

Elbadry, E.A. \& M.S.F. Tawfik. 1966. Life cycle of the mite Adactilidium sp. (Acarina: Pyemotidae), a predator of thrips eggs in the United Arab Republic. Ann. Entomol. Soc. Am. 59: 458-461.

Faroni, L.R.D’A., R.N.C. Guedes \& A.L. Matioli. 2000. Potential of Acarophenax lacunatus (Prostigmata: Acarophenacidae) as a biological control agent of Rhyzopertha dominica (Coleoptera: Bostrichidae). J. Stored Prod. Res. 36: 55-63.

Faroni, L.R.D’A., R.N.C. Guedes \& A.L. Matioli. 2001. Effect of temperature on development and population growth of Acarophenax lacunatus (Cross \& Krantz) (Prostigmata: Acarophenacidae) on Rhyzopertha dominica (F.) (Coleoptera: Bostrichidae). Biocontrol Sci. Technol. 11: 7-14.

Gao, J.R. \& P. Zou. 1994. A new species of Acarophenax (Acari: Acarophenacidae) from China. Entomotaxonomia 16: 291294.

Goldarazena, A., R. Jordana \& Z-Q. Zhang. 1997. Adactylidium moundi and $A$. costarricensis, two new species of Acarophenacidae (Acari: Tarsonemida) parasitic on Thysanoptera. Int. J. Acarol. 23: 261-268.

Goldarazena, A., R. Ochoa \& R. Jordana. 1999. Revision of the genus Paradactylidium Mahunka (Acari: Heterostigmata). Int. J. Acarol. 25: 91-99.

Goldarazena, A., R. Ochoa, R. Jordana \& B.M. Oconnor. 2001. Revision of the genus Adactylidium Cross (Acari: Heterostigmata: Acarophenacidae), mites associated with thrips (Thysanoptera). Proc. Entomol. Soc. Wash. 103: 473-516. 
Gonçalves, J.R., L.R.A. Faroni, R.N.C. Guedes \& C.R.F. de Oliveira. 2004. Insecticide selectivity to the parasitic mite Acarophenax lacunatus (Cross \& Krantz) (Prostigmata: Acarophenacidae) on Rhyzopertha dominica (Fabr.) (Coleoptera: Bostrichidae). Neotrop. Entomol. 33: 243-248.

Hagstrum, D.W., F.W. Flinn \& R.W. Howard. 1996. Ecology, p.71134. In B. Subramanyam \& D.W. Hagstrum (eds.), Integrated management of insects in stored products. New York, Marcel Dekker, 426p.

Hoschele, W. \& L.K. Tanigoshi. 1993. Pyemotes tritici (Acari: Pyemotidae), a potential biological control agent of Anagasta kuehniella (Lepidoptera: Pyralidae). Exp. Appl. Acarol. 17: 781-792.

Jandel Scientific. 1986. SigmaPlot Scientific Graphing Software - User's Manual. Jandel Scientific, San Rafael, CA, USA.

Kaliszewski, M., F. Athias-Binche \& E.E. Lindquist. 1995. Parasitism and parasitoidism in Tarsonemina (Acari: Heterostigmata) and evolutionary considerations. Adv. Parasitol. 35: 336-367.

Khaustov, A.A. 1999. Redescription of $<$ Pediculoides $>$ Ipidarius Redikortzev, 1947, and a description of a new species from the genus Paracarophenax (Acari: Heterostigmata: Acarophenacidae). Acarina 7: 57-59.

Kogan, M. \& J.R.P. Parra. 1981. Techniques and applications of measurements of consumption and utilisation of phytophagous insects, p.337-352. In G. Bhaskaran, S. Friedman \& J.C. Rodriguez (eds.), Current topics in insect endocrinology and nutrition. New York, Plenum, 362p.

Lindquist, E.E. 1983. Some thoughts on the potential for use of mites in biological control, including a modified concept of “parasitoids”, p.12-20. In M.A. Hoy, L. Knutson \& G.L. Cunningham (eds.), Biological control of pests of mites. University of California, Berkeley, 185p.

Livshitz, I.V. \& V.I. Mitranov. 1974. A new species of parasitic mite of the family Acarophenacidae (Acariformes) from Uzbekistan. Zool. Zh. 53: 288-289.

Magowski, W.L. 1994. Discovery of the first representative of the mite subcohort heterostigmata (Arachinida: Acari) in the Mesozoic Siberian amber. Acarologia 35: 229-241.

Mahunka, S. \& A. Fain. 1989. A new mite species of the cohort Tarsonemina (Acari: Acarophenacidae and Pygmephoridae). Parasit. Hung. 22: 125-136.

Mahunka, S. \& A.M. Zaki. 1991. Acarophenax rackae sp.n., a new mite species from the Egypt (Acari, Tarsonemina, Acarophenacidae). Parasit. Hung. 23: 121-127.

Maia, A.H.N., A.J.B. Luiz \& C. Camponola. 2000. Statistical inference on associated fertility table parameters using Jackknife technique: computational aspects. J. Econ. Entomol. 93: 511-518.
Marlatt, C.L. 1932. Report of the Chief Bureau of Entomology, Washington D.C., 1930-31: 87p. (source: Rev. Appl. Entomol. Serv. (A), 20: 215-218).

Newstead, R. \& H.M. Duvall. 1918. Bionomic, morfological and economic report on the acarids of stored grain and flour. R. Soc. Rep.Grain Pests (War) Comm. 2: 1-59.

Oliveira, C.R.F. de, L.R.D’A. Faroni \& R.N.C. Guedes. 2003a. Host egg preference by the parasitic mite Acarophenax lacunatus (Prostigmata: Acarophenacidae). J. Stored Prod. Res. 39: 571-575.

Oliveira, C.R.F. de, L.R.D’A. Faroni, R.N.C. Guedes \& A. Pallini. 2003b. Parasitism by the mite Acarophenax lacunatus on beetle pests of stored products. Biocontrol 48: 503-513.

Oliveira, C.R.F. de, L.R.D’A. Faroni, R.N.C. Guedes, A. Pallini \& J.R. Gonçalves. 2002. Parasitismo de Acarophenax lacunatus (Cross \& Krantz) (Prostigmata: Acarophenacidae) sobre Dinoderus minutus (Fabr.) (Coleoptera: Bostrichidae). Neotrop. Entomol. 31: 245-248.

Pedigo, L.P. \& M.R. Zeiss. 1996. Analyses in insect ecology and management. Ames, Iowa State University Press, 168p.

Rack, G. 1959. Acarophenax dermestidarum sp. n. (Acarina: Pyemotidae), ein Eiparasit von Dermestes Arten. Z. F. Parasitenkunde 19: 411-431.

Rady, G.H. 1992. New genus and species of Acarophenacidae (Acari: Tarsonemina) from Egypt. Ann. Agric. Sci. Moshtohor 30:1129-1135.

Rakha, M.A. \& M.M.H. Kandeel. 1983. Acarophenax meropsi n. sp., from the European bee eater, Merops apiaster in Egypt (Acari: Tarsonemida). Acarologia 24: 295-297.

SAS Institute. 1989. SAS/STAT User's guide for personal computers, version 6. SAS Institute, Cary, USA.

Steinkraus, D.C. \& E.A. Cross. 1993. Description and life history of Acarophenax mahunkai, n. sp. (Acari, Prostigmata: Acarophenacidae), an egg parasite of the lesser mealworm (Coleoptera: Tenebrionidae). Ann. Entomol. Soc. Am. 86: 239-249.

Wadhi, S.R. \& P. Kishore. 1975. New host record of Sciara hirtilineata (Sciaridae: Diptera) and Acarophenax tribolii Newstead and Duvall (Scutacaridae: Acarina). Indian J. Entomol. 35: 341-342.

Received 11/XI/05. Accepted 14/X/06. 